\title{
Can Endarterectomy Be Useful in Peripheral Arterial Occlusive Disease with Critical Limb Ischemia?
}

\author{
Binay Krishna Sarkar, Pares Banerjee \\ Department of Cardiovascular \& Thoracic surgery, Nil Ratan Sircar Medical College \& Hospital, Kolkata, West Bengal, India \\ Email: bappa_2k1_bk@yahoo.co.in
}

How to cite this paper: Sarkar, B.K. and Banerjee, P. (2019) Can Endarterectomy Be Useful in Peripheral Arterial Occlusive Disease with Critical Limb Ischemia? World Journal of Cardiovascular Surgery, 9, 163-169. https://doi.org/10.4236/wjcs.2019.911018

Received: June 5, 2019

Accepted: November 19, 2019

Published: November 22, 2019

Copyright (อ 2019 by author(s) and Scientific Research Publishing Inc. This work is licensed under the Creative Commons Attribution International License (CC BY 4.0).

http://creativecommons.org/licenses/by/4.0/

\section{(c) (i) Open Access}

\begin{abstract}
Importance of thrombendarterectomy (TEA) had declined with the advent of bypass techniques and availability of prosthetic grafts, in patients with Peripheral arterial occlusive disease (PAOD). Recently, there had been a significant shift towards lower limb revascularization using endoluminal techniques. However, previously available data evaluating the long leg bypass or combined endoluminal and bypass procedures have been too anatomically heterogeneous to be easily applied to patients with infrainguinal disease and tissue loss. Clinical decision making in Complex multilevel or diffuse peripheral arterial occlusive disease with multiple co-morbidities especially associated coronary arterial disease is challenging. We describe twelve patients of iliofemoral arterial occlusive disease with tibiopopliteal arterial occlusive disease along with multiple co-morbidities like coronary arterial disease and diabetes mellitus who showed marked improvement with minimal post-operative morbidities after iliofemoral or ileopopliteal bypass grafting with endarterectomy of the tibiopopliteal segment and related review of the literature.
\end{abstract}

\section{Keywords}

Peripheral Arterial Occlusive Disease, Coronary Arterial Disease, Thromboendarterectomy, Tibiopopliteal Disease

\section{Introduction}

Complex multilevel or diffuse peripheral arterial occlusive disease is a disabling condition where patients have chronic pain with or without ulceration or gangrene. Clinical decision making especially the choice of first-line intervention is often difficult especially in presence of tibiopopliteal disease in presence of mul- 
tiple co-morbid conditions especially coronary arterial disease. We have several procedures like endarterectomies, bypass grafting and endoluminal procedures often used in combination to address these complex lesions. Thromboendarterectomy (TEA) was the procedure of choice for patients with Peripheral arterial occlusive disease (PAOD) [1] [2]. With the advent of bypass techniques and availability of prosthetic grafts, there is a marked decline in the number of Surgeons performing this technically demanding procedure [1]. But there are advantages of autogenously vascular reconstruction and it is useful as an adjunctive procedure.

In 1946 Portugese Surgeon Dos Santos did first TEA through 2 arterectomies and he called it as disobliteration [1]. Leriche termed removal of obstructing thrombus as well as diseased arterial intima as Thromboendarterectomy (TEA). It has been shown that excellent results can be achieved when the technique was precisely performed especially in popliteal artery segments [2]. Almost all anatomic patterns of occlusive atherosclerosis, whether localized, diffuse, degenerative, or occlusive are amenable to endarterectomy. The atherosclerotic plaques are localized to the intima and inner media of the diseased arterial wall. A cleavage plane can be developed between the diseased and non-diseased portions of the arterial wall. When properly performed the diseased inner media and intima can easily be separated from rest of the arterial wall.

Over the last few years there has been a significant shift towards lower limb revascularization using endoluminal techniques [3]. However, in many instances endoluminal techniques alone are unable to salvage limbs that exhibit tissue loss. Many of these patients do not have adequate conduit for a long leg bypass, while tibial angioplasty does not appear to restore adequate perfusion to heal many significant foot lesions, making combined procedures less attractive. However, previously available data evaluating combined endoluminal and bypass procedures have been too anatomically heterogeneous to be easily applied to patients with infrainguinal disease and tissue loss.

Whether a particular procedure is better than the other in influencing decisions for the choice of first-line therapy is difficult. Patient-specific factors like site, extent and complexity of the lesion, comorbidities and durability of outcome of an intervention are critical in planning an intervention. In multilevel arterial occlusion especially with involvement of tibio-popliteal segment made endarterectomy a viable option to address these cases with complex arterial occlusive disease in our series.

\section{Methods}

A retrospective study of twelve consecutive patients with complex arterial occlusive disease with involvement of tibio-popliteal segment is made in our institute of Nilratan Sircar Medical College who were operated over a period of eleven years from 2009 to 2018 and tibiopopliteal endarterectomy was done in all these cases in addition to bypass grafting. Cases with Femoropopliteal disease involving only proximal popliteal artery were excluded from the study. Transatlantic Inter-society Consensus (TASC) classification of aorto-iliac and femoral popli- 
teal lesions was followed during selection of patients. Demographic data, comorbidities, laboratory data, angiographic anatomic data and clinical outcome were recorded.

\section{Results}

All these symptomatic patients underwent prosthetic bypass grafting along with poplliteal endarterectomy. All these patients had extensive popliteal disease. Of these twelve patients, all (100\%) patients also had significant posterior tibial disease, four (25\%) patients had anterior tibial disease and two (16.6\%) patients had calcified disease involving trifurcation and three patients required venous patch plasty to augment the tibiopopliteal junction. Distal landing zone of the prothetic grafts was chosen carefully in the popliteal fossa on the popliteal artery having adequate lumen. Nine cases underwent femoropopliteal bypass grafting and ileopopliteal bypass grafting were done along with patchplasty in three cases. Transobturator route was used in three cases to avoid inguinal infection.

\section{Endarterectomy:}

In the operation theatre, the patient was positioned prone and was operated under spinal anesthesia. An incision was made behind the left knee which was extended downwards in the mid part of the leg. Deep fascia was incised and gastrocnemi, popliteus muscles were retracted to expose the lower part of the popliteal artery and soleus muscle.

Popliteal vessles were carefully dissected from the fatty tissue in the popliteal fossa and popliteal artery was found to be cord like. Soleus muscle was carefully manipulated and carefully dissected to expose the trifurcation of the popliteal artery preserving the soleal venous plexus. The popliteal artery and the posterior tibial artery were found to be of good caliber at this region but were cord like in majority of our cases. Endarterectmy was planned and the popliteal artery was longitudinally incised near the trifurcation of the popliteal artery after injecting heparin intravenously at the dose of $1 \mathrm{mg}$ per kg of body weight (Figure 1(a)). The involved popliteal artery and proximal tibial artery were carefully desobliterated after carefully and precisely developing a plane in between diseased and non-diseased part of the arterial wall with the help of fine instruments like ring tip forceps, mosquito artery forceps and fine right angled forceps.

A long endarterectomy specimen (Figure 1(b)) could be retrieved from the popliteal artery and the posterior tibial artery by precise application of traction and counter traction till the end points and there was free flow of blood from the arterial lumens both from above and from below the incision. The incision over the artery was closed with 6 " 0 " polypropylene suture or patch plasty was done and the hemostasis was achieved. Wound was closed after putting Romosuction drain after the bypass procedure (Table 1 ).

\section{Postoperative period:}

The hemodynamics of the patients was stable postoperatively. None of them required ventilator support. None of them required emergency reoperation. 
Table 1. Showing demographic data, associated morbidity, procedures and outcome in the patient population.

\begin{tabular}{|c|c|c|}
\hline Total number $(n=12)$ & & \\
\hline Sex & Male $(10,83.3 \%)$ & Female $(2,16.6 \%)$ \\
\hline Age (years) range (mean) & $45-70(61)$ & $56-64(60)$ \\
\hline Hypertension & $10(100 \%)$ & $2(100 \%)$ \\
\hline Diabeted Mellitus & $6(60 \%)$ & $2(100 \%)$ \\
\hline Hyperlipidemia & $10(100 \%)$ & $2(100 \%)$ \\
\hline COPD & $3(30 \%)$ & $1(50 \%)$ \\
\hline Coronary arterial disease (mild) & $9(90 \%)$ & $2(100 \%)$ \\
\hline Renal insufficiency & 0 & 0 \\
\hline Active smoking & $10(100 \%)$ & 0 \\
\hline \multicolumn{3}{|l|}{ Number of tibial vessels involved } \\
\hline 0 & 0 & 0 \\
\hline 1 & 5 & 1 \\
\hline 2 & 3 & 1 \\
\hline 3 & 2 & 0 \\
\hline Venous patch plasty & $2(20 \%)$ & $1(50 \%)$ \\
\hline Ileopopliteal bypass & $2(20 \%)$ & $1(50 \%)$ \\
\hline Femoropopliteal bypass & $8(80 \%)$ & $1(50 \%)$ \\
\hline Transobturator bypass & $2(20 \%)$ & $1(50 \%)$ \\
\hline Both leg bypass & $3(30 \%)$ & 0 \\
\hline \multicolumn{3}{|l|}{ Post-op complication } \\
\hline 1) Wound infection & $4(40 \%)$ & $1(50 \%)$ \\
\hline 2) Delayed healing & $2(20 \%)$ & 0 \\
\hline 3) Reoperation & 0 & 0 \\
\hline 4) Bleeding & $1(10 \%)$ & 0 \\
\hline Patency (at the end of 1 year) & $10(100 \%)$ & $2(100 \%)$ \\
\hline
\end{tabular}

There was no major bleeding episodes or renal insufficiency. Postoperatively peripheral pulses were palpable in the leg and there was symptomatic improvement (Figures 2(a)-(c)) in all cases. Milld bleeding due to anticoagulation was noted in one patient. Wound infection ranging from hyperemia to discharge was noted in five (41.6\%) patients and all were diabetic. This indicates the tendency of the diabetic patients to develop wound infections and microvascular disease might be responsible for this. However, every effort needs to be made to control diabetes in the perioperative period. Of these 5 patients two (16.6\%) developed superficial wound disruptions and delayed healing. All these patients were followed up in the outpatient department and peripheral angio was done at the end of one year. All patients showed patency. Two patients lost to follow up after 6 years. Maximum follow-up was up to nine years. 


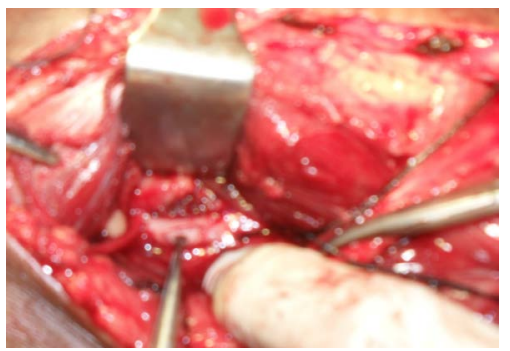

(a)

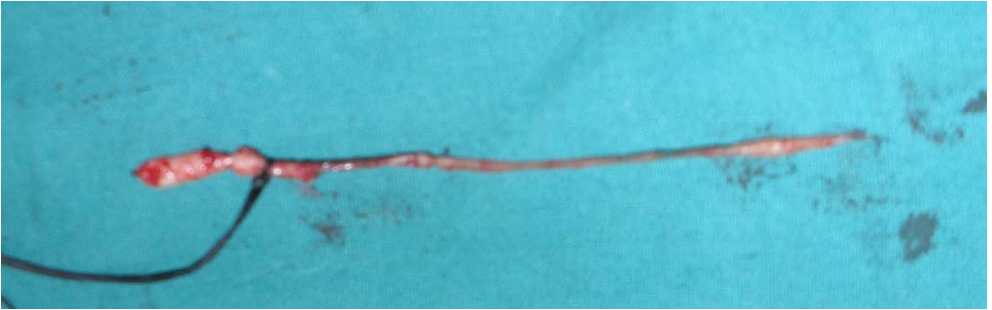

(b)

Figure 1. (a) peroperative picture showing endarterectomy in progress; (b) the picture shows endarterectomy specimen.

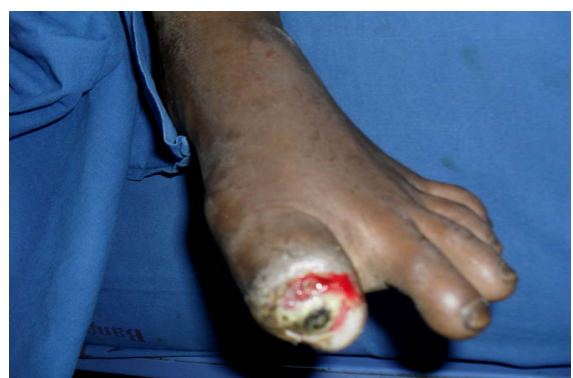

(a)

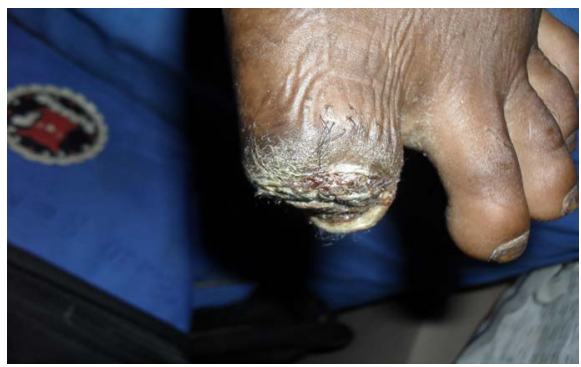

(b)

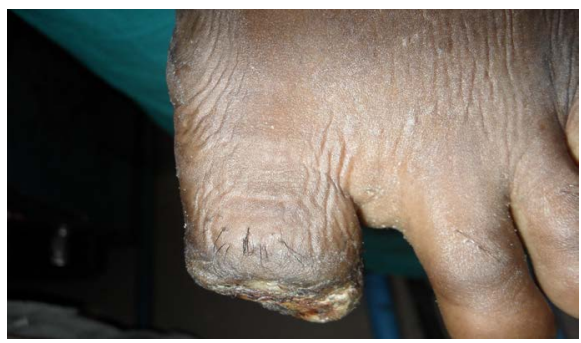

(c)

Figure 2. (a) postop day 1, picture of the great toe wound; (b) great toe wound at the 3rd postop day; (c) great toe wound at the 7 th postop day. 


\section{Discussion}

Peripheral arterial disease correlates strongly with risk of major cardiovascular events and they have a high prevalence of coexistent coronary and cerebrovascular disease [1] [2] [3] [4]. Because the prevalence of PAD increases progressively with age, it is a major health problem in elderly.

Thromboendarterectomy (TEA) was more frequently performed in patients with Peripheral arterial occlusive disease (PAOD) until the introduction of bypass techniques and availability of prosthetic grafts. TEA had been discouraged due to perioperative thrombosis, increased morbidity and rate of amputation [4]. The poor results probably contributed to improper selection and perioperative management of these patients, improper tissue handling and healing after the procedure.

Endovascular practice, percutaneous transluminal angioplasty with or without stenting, is used far more frequently for all types of lower extremity occlusive lesions, reflecting the continuing advances in imaging techniques, angioplasty equipment, and endovascular expertise [1]. The role of endovascular intervention in the treatment of limb-threatening ischemia is also expanding, and its promise of limb salvage and symptom relief with reduced morbidity and mortality makes percutaneous transluminal angioplasty/stenting an attractive alternative to surgery.

Recently many authors had established the role of endarterectomy in chronic critical limb ischemia especially in combination with other procedures in multilevel arterial obstructive disease [3] [4] [5]. The incidence of high-risk patients suffering from critical limb ischemia due to multilevel arterial obstructive disease is growing rapidly. Invasive surgical procedures to restore inflow to the crural and pedal circulation had substantial morbidity and mortality. On the other hand, the mid-term and long-term outcomes of sole percutaneous revascularization procedures are disappointing for similar lesions. Hybrid endovascular and open surgical revascularization procedures might be of benefit because of its less invasive character, no need for extensive venous graft material, and the ability to overcome long-segment arterial obstructions [4]. However, diabetes, renal insufficiency, and chronic limb ischaemia could compromise its long-term patency.

The effectiveness of TEA is confirmed in the symptomatic improvement and salvage of the arm, and the lower costs, when the indications and the accuracy of surgical technique are respected [6]. The advantages it offers for the surgical treatment of steno-obstructive lesions of aortoiliac and femoro-popliteal regions cannot be overemphasized.

In patients with limb ischemia, the quality of life may be improved by physical training, vasoactive medicaments and optimal management of concomitant diseases [7]. The role of platelets and the importance of platelet inhibition are well established in patients with vascular disease. Aspirin and in second priority ticlopidine are suited for secondary prevention of arteriosclerosis not only in the 
extracranial, but also in the peripheral vascular region. After endarterectomy and catheter therapy, aspirin improves the long-term outcome by reducing the incidence of restenoses. Better results are obtained by oral anticoagulation in patients with emboli and after local thrombolysis. Various pharmalogic agents may be used to facilitate healing and therefore improve the results of endarterectomy like heparin, ACE inhibitors and growth factor antagonists.

In our series, majority of our patients were elderly diabetic with stable ischaemic heart disease and tissue loss at presentation with other co-morbidities and could be operated under spinal anesthesia with minimal postoperative morbidities. These patients remained pain free and showed good long term results.

Because of low cost, use of autogenous vascular reconstruction, no prosthetics use, low risk of infection, less morbidities, TEA will assume increasingly important role in complex peripheral arterial occlusive disease. Further study with more number of patients and long term follow-up would be required to see its long-lasting benefit.

\section{Conflicts of Interest}

The authors declare no conflicts of interest regarding the publication of this paper.

\section{References}

[1] Allaqaband, S., Kirvaitis, R., Jan, F. and Bajwa, T. (2009) Endovascular Treatment of Peripheral Vascular Disease. Current Problems in Cardiology, 34, 359-476. https://doi.org/10.1016/j.cpcardiol.2009.05.001

[2] Smeets, L. et al. (2003) Remote Endarterectomy: First Choice in Surgical Treatment of Long Segmental SFA Occlusive Disease? European Journal of Vascular and Endovascular Surgery, 25, 583. https://doi.org/10.1053/ejvs.2002.1921

[3] Lantis, J., Jensen, M., Benvenisty, A., Mendes, D., Gendics, C. and Todd, G. (2008) Outcomes of Combined Superficial Femoral Endovascular Revascularization and Popliteal to Distal Bypass for Patients with Tissue Loss. Annals of Vascular Surgery, 22, 366-371. https://doi.org/10.1016/j.avsg.2007.09.016

[4] Schrijver, A.M., Moll, F.L. and De Vries, J.P. (2010) Hybrid Procedures for Peripheral Obstructive Disease. The Journal of Cardiovascular Surgery (Torino), 51, 833-843.

[5] Al-Khoury, G., Marone, L., Chaer, R., Rhee, R., Cho, J., Leers, S., Makaroun, M. and Gupta, N. (2009) Isolated Femoral Endarterectomy: Impact of SFA TASC Classification on Recurrence of Symptoms and Need for Additional Intervention. Journal of Vascular Surgery, 50, 784-789. https://doi.org/10.1016/j.jvs.2009.05.053

[6] Troilo, D., Marchionni, F. and Quadrini, N. (1997) Current Role of Thrombendarterectomy in Chronic Peripheral Obstructive Arteriopathy. Minerva Cardioangiologica, 45, 547-552.

[7] Bollinger, A. (1993) Treatment Strategies in Arterial Occlusive Diseases. Schweizerische Rundschau fur Medizin Praxis, 82, 1354-1358. 